\title{
A note on stability in dual-phase-lag heat conduction
}

\author{
RAMÓN QUINTANILLA ${ }^{1}$ \\ Department of Applied Mathematics II \\ UPC Terrassa, Colom 11, 08222 Terrassa, Spain \\ ramon.quintanilla@upc.edu \\ REINHARD RACKE ${ }^{2}$ \\ Department of Mathematics and Statistics \\ University of Konstanz \\ 78457 Konstanz, Germany \\ reinhard.racke@uni-konstanz.de
}

\begin{abstract}
In this note we compare two different mathematical hyperbolic models in dual-phaselag heat conduction proposed by Tzou, and we ask for the parameter regions where stability can be expected. It is demonstrated that the parameter regions for the two lag-parameters $\tau_{q}$ and $\tau_{\theta}$ are different for the two models. That is, for certain parameters, in one model stability is expected while for the other one it is known that it is not stable. The first apparent contradiction is contrasted with the fact that known values for real materials (several metals are considered here) are in a range where both models predict stability or non-stability, respectively. Still, as a conclusion, one model should be considered only in a restricted parameter region.
\end{abstract}

\section{Introduction}

There are several hyperbolic theories of heat conduction, also called theories of second sound, where the propagation of heat is modeled with finite propagation speed, in contrast to the classical model using Fourier's law leading to infinite propagation speed of heat signals, see the survey by Chandrasekharaiah [1] or the books of Müller and Ruggeri [4] and Jou et al. [3].

In 1995, Tzou [11] proposed a theory of heat conduction,

$$
\theta_{t}+\operatorname{div} \mathbf{q}=0
$$

${ }^{0}$ AMS subject classification: 35 L 35, 80 A 20

Keywords and phrases: hyperbolic models in heat conduction, stability

${ }^{1}$ Supported by the project "Aspects of stability in thermomechanics" (BFM/FEDER 2003-00309)

${ }^{2}$ Supported by the DFG-project "Hyperbolic Thermoelasticity" (RA 504/3-1). 
with temperature $\theta$ and heat flux vector $\mathbf{q}$, in which the Fourier law is replaced by an approximation of the equation

$$
\mathbf{q}\left(\mathbf{x}, t+\tau_{q}\right)=-k \nabla \theta\left(\mathbf{x}, t+\tau_{\theta}\right), \quad \tau_{q}>0, \tau_{\theta}>0
$$

where $k>0$, and $\tau_{q}$ is the phase-lag of the heat flux and $\tau_{\theta}$ is the phase-lag of the gradient of the temperature. The relation (1.2) states that the gradient of temperature at a point in the material at time $t+\tau_{\theta}$ corresponds to the heat flux vector at the same point at time $t+\tau_{q}$. The delay time $\tau_{\theta}$ is caused by microstructural interactions such as phonon scattering or phonon-electron interactions. The delay $\tau_{q}$ is interpreted as the relaxation time due to fast-transient effects of thermal inertia. The corresponding thermoelastic model was proposed in [1]. Instead of Fourier's law, being equivalent to assuming

$$
\tau_{q}=\tau_{\theta}=0
$$

and leading to the classical parabolic equation of heat conduction together with the physical paradoxon of infinite propagation speed, we consider the model proposed by Tzou [11], where a second-order approximation for $\mathbf{q}$ and a first-order approximation for $\theta$ is used, turning (1.2) into

$$
\mathbf{q}+\tau_{q} \mathbf{q}_{t}+\frac{\tau_{q}^{2}}{2} \mathbf{q}_{t t}=-k \nabla \theta-k \tau_{\theta} \nabla \theta_{t}
$$

and hence turning (1.1) into the following hyperbolic equation for the temperature:

$$
\frac{\tau_{q}^{2}}{2} \theta_{t t t}+\tau_{q} \theta_{t t}+\theta_{t}=k \Delta \theta+k \tau_{\theta} \Delta \theta_{t}
$$

In this note, we discuss all the possibilities proposed, up to now, for the dual-phase-lag heat conduction theory. The equation (1.3) is the Taylor expansion of the equation (1.1) up to the first order with respect to the parameters $\tau_{\theta}$ and up to the second order with respect to $\tau_{q}$. This derivation produces a hyperbolic equation. We note that in the absence of the supply terms, equation (1.4) agrees (see [12], p.3234) with the hyperbolic equation obtained for the two-step radiation heating model proposed by Qiu and Tien [6].

A natural question is the determination of the time parameters $\tau_{q}$ and $\tau_{\theta}$. We believe that mathematical analysis could help to reveal several conditions on the parameters. One condition to be satisfied by a heat conduction equation is the stability of its solutions. We recall that a system is stable if solutions are bounded for all times. Otherwise, we say that the system is unstable. That is the solution becomes unbounded when time increases. Therefore, we expect that the parameters which define a heat equation must be such that the system is stable. This note is addressed to clarify this aspect in the case of the dual-phase-lag theories. 
For the equation (1.4), together with appropriate initial and boundary conditions, Quintanilla [7] has shown that the system is (even exponentially) stable if

$$
x:=\frac{\tau_{\theta}}{\tau_{q}}>\frac{1}{2}
$$

holds, and that it is not (exponentially) stable if

$$
x<\frac{1}{2}
$$

holds. The case $x=1$ is not discussed explicitly in [7] but is expected to lead to exponential stability, cp. Section 2. The exponential stability has also been shown for onedimensional corresponding systems of dual-phase-lag thermoelasticity, see the author's papers $[8,9]$.

As Tzou notes in his paper [12, p. 3237]: From a consistent mathematical point of view, the second-order expansions in $\tau_{\theta}$ and $\tau_{q}$ should also involve a second-order time derivative in $\tau_{\theta}^{2}$... resulting in an equation of parabolic type.

Thus, taking second-order approximations both for the temperature and the heat flux, we have to replace (1.3) by

$$
\mathbf{q}+\tau_{q} \mathbf{q}_{t}+\frac{\tau_{q}^{2}}{2} \mathbf{q}_{t t}=-k \nabla \theta-k \tau_{\theta} \nabla \theta_{t}-k \frac{\tau_{\theta}^{2}}{2} \nabla \theta_{t t}
$$

which turns (1.1) into the following equation for the temperature:

$$
\frac{\tau_{q}^{2}}{2} \theta_{t t t}+\tau_{q} \theta_{t t}+\theta_{t}=k \Delta \theta+k \tau_{\theta} \Delta \theta_{t}+k \frac{\tau_{\theta}^{2}}{2} \Delta \theta_{t t}
$$

For the two equations (1.4) and (1.8) we shall compare the possible regions for $\left(\tau_{\theta}, \tau_{q}\right)$ to yield stability. We shall see that they are essentially different, that is, there is an open set of parameter values for which in one system stability is expected while in the other it has been proved that there is no stability.

As a consequence of a unique physical behavior, for real materials with these parameter values, only one of the models can be justified. Fortunately, it turns out that for classes of materials as metals like copper, silver, gold or lead, for which the parameter values have been determined, the values are in the set where both models predict the same.

The paper is organized as follows: In Section 2 we review the results by Quintanilla [7] on (exponential) stability for the system (1.4) in terms of the eigenvalues of the characteristic polynomial. The investigation of the system (1.8), also in terms of the eigenvalues, is done in Section 3. The comparison is presented in Section 4. 


\section{The model (1.4) revisited}

In [7] it was shown by energy methods that the system governed by the differential equation (1.4) for Dirichlet boundary conditions is exponentially stable provided (1.5) holds, and, using the Hurwitz criterion, that it is not exponentially stable if (1.6) holds.

The conditions (1.5), (1.6) can be easily understood looking, as in [7, Sec. 5], at the roots of the characteristic polynomial associated to (1.4), where we denote by $\left(\lambda_{n}\right)_{n}$ the eigenvalues of the Laplace operator $-\Delta$ for Dirichlet boundary conditions in a bounded reference configuration $\Omega$ :

$$
\beta^{3}+\frac{2}{\tau_{q}} \beta^{2}+\frac{2+2 k \tau_{\theta} \lambda_{n}}{\tau_{q}^{2}} \beta+\frac{2 k \lambda_{n}}{\tau_{q}^{2}}=0 .
$$

That is, if we look for solutions of the equation (1.4) of the form $\exp (\beta t) \Phi_{n}(\mathbf{x})$, where $\Phi_{n}$ is the eigenfunction to the eigenvalue $\lambda_{n}$ of the problem

$$
\Delta \Phi_{n}+\lambda_{n} \Phi_{n}=0, \text { in } \Omega, \quad \Phi_{n}=0, \text { on } \partial \Omega,
$$

then $\beta$ satisfies the equation (2.1), and purely imaginary $\beta=i \alpha, \alpha$ real, would express oscillatory behavior.

By the Hurwitz criterion, all three roots of the polynomial

$$
\beta^{3}+l_{1} \beta^{2}+l_{2} \beta+l_{3}=0
$$

have negative real parts if and only if

$$
l_{j}>0, j=1,2,3, \quad l_{1} l_{2}>l_{3}
$$

holds. For (2.1) this conditions turns into

$$
\lambda_{n} k\left(\frac{2 \tau_{\theta}}{\tau_{q}}-1\right)+\frac{2}{\tau_{q}}>0
$$

which is not satisfied uniformly in $\lambda_{n}$ if and only if (1.6) holds. That is, we have recalled the characterization of exponential stability from [7].

We remark that the discussion of the eigenvalues and looking for the supremum of the real parts to be strictly less than zero, is in general not sufficient for the corresponding system (semigroup) to be exponentially stable. For infinite dimensional systems, in contrast to ordinary differential equations, the supremum of the spectrum does not describe the so-called type of the semigroup, cp. the discussion in $[5,10]$ and the references therein. But for appropriate boundary conditions, it can be shown by special analysis, as for thermoelasticity with second sound under the Cattaneo model, see [2], that the type of the semigroup is really determined by the supremum of the real parts of the spectrum. Our discussion of stability in the next sections continues in the discussion of the spectrum. 


\section{The model (1.8)}

Now we turn to the second model represented by the differential equation (1.8). The characteristic polynomial now reads as

$$
\beta^{3}+\left(\frac{2}{\tau_{q}}+\frac{k \tau_{\theta}^{2} \lambda_{n}}{\tau_{q}^{2}}\right) \beta^{2}+\left(\frac{2}{\tau_{q}^{2}}+\frac{2 k \tau_{\theta} \lambda_{n}}{\tau_{q}^{2}}\right) \beta+\frac{2 k \lambda_{n}}{\tau_{q}^{2}}=0,
$$

and by the criterion (2.2) the following inequality has to hold uniformly in $\lambda_{n}$ :

$$
\lambda_{n}^{2} \underbrace{\left[\frac{k^{2} \tau_{\theta}^{3}}{\tau_{q}^{2}}\right]}_{=: A}+\lambda_{n} \underbrace{\left[k\left(\frac{\tau_{\theta}^{2}}{\tau_{q}^{2}}-1+\frac{2 \tau_{\theta}}{\tau_{q}}\right)\right]}_{=: B}+\underbrace{\frac{2}{\tau_{q}}}_{=: C}>0 .
$$

Since $A$ and $C$ are strictly positive, a sufficient condition is to have $B \geq 0$ which turns into

$$
\frac{\tau_{\theta}}{\tau_{q}} \geq \sqrt{2}-1
$$

which is already a condition being different from (1.5) and gives a larger expectation for stability since $\sqrt{2}-1<\frac{1}{2}$.

Now let $B<0$, i.e. let $x<\sqrt{2}-1$. Then we expect stability if

$$
f(y):=A y^{2}+B y+C>0
$$

uniformly in $\lambda_{n}$, i.e. if the largest zero (if there is any real zero at all) of the polynomial $f$ is less than the smallest eigenvalue $\lambda_{1}$.

Case I:

$$
B^{2}<4 A C
$$

In this case $f$ does not have real zeros, hence (3.4) is satisfied. The condition (3.5) is equivalent to

$$
g(x):=x^{4}-4 x^{3}+2 x^{2}-4 x+1<0
$$

where $x$ denotes as before the ration $\frac{\tau_{\theta}}{\tau_{q}}$. The zeros of $g$ as a polynomial in $x$ are

$$
\tilde{x}_{1}=\mathrm{i}, \quad \tilde{x}_{2}=-\mathrm{i}, \quad \tilde{x}_{3}=2+\sqrt{3}, \quad \tilde{x}_{4}=2-\sqrt{3} .
$$

Hence, in view of (3.5), $x$ has to satisfy

$$
2-\sqrt{3}<x<2+\sqrt{3} .
$$

That is, for values $\left(\tau_{\theta}, \tau_{q}\right)$ with

$$
x>2-\sqrt{3}
$$

we are already in the expected region of stability. 


\section{Case II:}

$$
B^{2} \geq 4 A C
$$

In this case we have to consider the region where $x \leq 2-\sqrt{3}$, and to see when the largest positive zero of $f$ is less than $\lambda_{1}$. This is equivalent to

$$
-B+\sqrt{B^{2}-4 A C}<2 A \lambda_{1}
$$

or

$$
B^{2}-4 A C<\left(2 A \lambda_{1}+B\right)^{2}
$$

or, equivalently again,

$$
x^{4}-4 x^{3}+2 x^{2}-4 x+1<\left(x^{2}\left(1+2 k \tau_{\theta} \lambda_{1}\right)+2 x-1\right)^{2} .
$$

Hence

$$
\left.h(x):=x^{2}\left(\left(2 k \tau_{\theta} \lambda_{1}\right)^{2}+4 k \tau_{\theta} \lambda_{1}\right)\right)+8\left(1+k \tau_{\theta} \lambda_{1}\right) x-4 k \tau_{\theta} \lambda_{1}>0
$$

is a necessary and sufficient condition. The zeros $\hat{x}_{1 / 2}$ of $h$ as a polynomial in $x$ are

$$
\hat{x}_{1 / 2}=\frac{ \pm \sqrt{\left(1+k \tau_{\theta} \lambda_{1}\right)^{2}+\left(k \tau_{\theta} \lambda_{1}\right)^{2}+\left(k \tau_{\theta} \lambda_{1}\right)^{3}}-\left(1+k \tau_{\theta} \lambda_{1}\right)}{k \tau_{\theta} \lambda_{1}\left(1+k \tau_{\theta} \lambda_{1}\right)} .
$$

Since $\hat{x}_{2}<0$ we obtain from (3.9) the following condition on $x$ :

$$
x>\frac{\sqrt{\left(1+k \tau_{\theta} \lambda_{1}\right)^{2}+\left(k \tau_{\theta} \lambda_{1}\right)^{2}+\left(k \tau_{\theta} \lambda_{1}\right)^{3}}-\left(1+k \tau_{\theta} \lambda_{1}\right)}{k \tau_{\theta} \lambda_{1}\left(1+k \tau_{\theta} \lambda_{1}\right)} .
$$

This condition can be a real restrictive condition, e.g. for $\tau_{q}$ being large in comparison to $\tau_{\theta}$. On the other hand, the right-hand side of (3.10) tends to zero if $\lambda_{1}$ tends to zero, as well as if $\lambda_{1}$ tends to infinity. This means, that for a fixed material, the condition (3.10) is satisfied if the domain is small or large enough, since the smallest eigenvalue depends on the size of the domain. For example, in one space dimension, if the domain is the interval $(0, L)$ with $L>0$, one has

$$
\lambda_{n}=\frac{n^{2} \pi^{2}}{L^{2}}
$$

That is, for a fixed material we have

$$
\exists l_{0}, L_{0}>0 \quad \forall L \notin\left[l_{0}, L_{0}\right]: \quad(3.10) \text { holds. }
$$

We remark that a condition of the last type is known e.g. for equations of the type

$$
w_{t}-w_{x x}-\mu w=0, \quad \text { in }(0, \infty) \times(0, L),
$$


where $\mu>0$, and for Dirichlet boundary conditions. We have (exponential) stability if and only if

$$
\mu<\lambda_{1}=\frac{\pi^{2}}{L^{2}}
$$

Summarizing the considerations of this section, we are in the regime of stability if

$$
x=\frac{\tau_{\theta}}{\tau_{q}}>2-\sqrt{3}
$$

or if

$$
x \leq 2-\sqrt{3} \text { and (3.10) holds, }
$$

and the condition (3.10) is for one-dimensional domains $(0, L)$ - for fixed $\left(\tau_{\theta}, \tau_{q}\right)$ satisfied if $L$ is small enough or large enough, respectively; more generally in higher dimensions: if the first eigenvalue of the Dirichlet Laplacian is small or large enough, respectively.

Obviously, the characterization of the stability region is quite different from that of the model discussed in the previous section. A comparison and conclusions for the modelling and for real materials is following.

\section{Comparisons}

As shown in the last two sections there are regions, for which the model (1.4) predicts non-stability while the model (1.8) predicts stability, for example (but not only) if

$$
2-\sqrt{3}<x<\frac{1}{2}
$$

Since the physical behavior is either stable or not, only one of the model can describe the situation correctly. This problem becomes a void issue for applications only if for real materials one is always in the region where both models predict the same behavior.

From the work of Tzou [12] (in [11] the values are given slightly different, but this does not affect the reasoning), we take the following values for $\tau_{\theta}$ and $\tau_{q}$, measured in picoseconds, for the metals copper, silver, gold and lead.

\begin{tabular}{|c|c|c|}
\hline & $\tau_{\theta}$ & $\tau_{q}$ \\
\hline $\mathrm{Cu}$ & 70.833 & 0.4648 \\
$\mathrm{Ag}$ & 89.286 & 0.7838 \\
$\mathrm{Au}$ & 89.286 & 0.7838 \\
$\mathrm{~Pb}$ & 12.097 & 0.1720 \\
\hline
\end{tabular}


For the four metals the ratio $x$ of the lag-parameters is (much) larger than $\frac{1}{2}$, i.e. we are not in any ambiguous region. Thus for these examples both models are fine. Not knowing the ratio $x$ for all materials, it might be useful to keep the characterizations of our paper in mind. Moreover, for numerical calculations the limiting regions of stability are also good to know.

Finally, we remark that we can also compare the two models above with the situation where a) both for the heat flux and for the temperature just a first-order approximation is used:

$$
\mathbf{q}+\tau_{q} \mathbf{q}_{t}=-k \nabla \theta-k \tau_{\theta} \nabla \theta_{t},
$$

or where b) we take a first-order approximation for the heat flux, but a second-order approximation for the temperature, i.e.

$$
\mathbf{q}+\tau_{q} \mathbf{q}_{t}=-k \nabla \theta-k \tau_{\theta} \nabla \theta_{t}-k \frac{\tau_{\theta}^{2}}{2} \nabla \theta_{t t}
$$

In case a) the equation for $\theta$ resulting from (1.1), (4.2) is

$$
\tau_{q} \theta_{t t}+\theta_{t}=k \Delta \theta+k \tau_{\theta} \Delta \theta_{t}
$$

It is worth recalling several recent contributions to this equation (see $[13,14,15])$. Here the characteristic polynomial to this equation of viscoelastic type

$$
\beta^{2}+\frac{1+k \tau_{\theta} \lambda_{n}}{\tau_{q}} \beta+\frac{k \lambda_{n}}{\tau_{q}}
$$

has two roots

$$
\beta_{1 / 2}=\frac{ \pm \sqrt{\left(1+k \tau_{\theta}\right)^{2}-4 \tau_{q} k \lambda_{n}}-\left(1+k \tau_{\theta}\right)}{2 \tau_{q}}
$$

which have real parts being negative, uniformly in $\lambda_{n}$. That is, no condition arises on the lag-parameters for stability.

In case b) we obtain for $\theta$ the differential equation

$$
\tau_{q} \theta_{t t}+\theta_{t}=k \Delta \theta+k \tau_{\theta} \Delta \theta_{t}+k \frac{\tau_{\theta}}{2} \Delta \theta_{t t}
$$

with characteristic polynomial

$$
\beta^{2}+\frac{2\left(1+k \tau_{\theta} \lambda_{n}\right)}{2 \tau_{q}+k \tau_{\theta}^{2} \lambda_{n}} \beta+\frac{2 k}{2 \tau_{q}+k \tau_{\theta}^{2} \lambda_{n}}
$$

with two zeros

$$
\beta_{1 / 2}=\frac{ \pm \sqrt{\left(1+k \tau_{\theta} \lambda_{n}\right)^{2}-2 k\left(2 \tau_{q}+k \tau_{\theta}^{2} \lambda_{n}\right)}-\left(1+k \tau_{\theta} \lambda_{n}\right)}{2 \tau_{q}+k \tau_{\theta}^{2} \lambda_{n}}
$$

that are also have negative real parts which are bounded away from zero uniformly in $\lambda_{n}$. That is, again we do not have any restriction for the ratio of the lag-parameters. 


\section{Conclusion}

In this short note we have analyzed the range of the parameters $\tau_{\theta}$ and $\tau_{q}$ for different kind of approximations in the dual-phase-lag theory in order to guarantee that the solutions of the corresponding heat equation are stable. We have seen that:

1. When we approximate until first order in $\tau_{q}$ and until first or second order in $\tau_{\theta}$, the system is always stable.

2. When we approximate until second order in $\tau_{q}$ and only until first order in $\tau_{\theta}$, the system is stable if $\tau_{\theta} / \tau_{q}>1 / 2$ and unstable if $\tau_{\theta} / \tau_{q}<1 / 2$.

3. When we approximate until second order both in $\tau_{\theta}$ and in $\tau_{q}$, the system is stable if $\tau_{\theta} / \tau_{q}>2-\sqrt{3}$. If $\tau_{\theta} / \tau_{q}<2-\sqrt{3}$ and the quotient $x=\tau_{\theta} / \tau_{q}$ satisfies condition (3.10), we are also in the the stability regime.

4. Whenever $\tau_{\theta} / \tau_{q}>1 / 2$, the several models predict the same behavior.

\section{References}

[1] DS. Chandrasekharaiah, Hyperbolic thermoelasticity: A review of recent literature, Appl. Mech. Rev., 51(1998), pp. 705-729.

[2] T. Irmscher, R. Racke, Sharp decay rates in parabolic and hyperbolic thermoelasticity, submitted to: IMA J. Appl. Math.

[3] D. Jou, J. Casas-Vazquez, G. Lebon, Extended Irreversible Thermodynamics, SpringerVerlag, Berlin, 1996.

[4] I. Müller, T. Ruggeri, Rational and Extended Thermodynamics, Springer-Verlag, NewYork, 1998.

[5] J. Prüss, On the spectrum of $C_{0}$-semigroups, Trans. AMS, 284(1984), pp. 847-857.

[6] T.Q. Qiu, C. L. Tien, Heat transfer mechanisms during short-pulse laser heating of metals, ASME J. Heat Transfer, 115(1993), pp. 835-841.

[7] R. Quintanilla, Exponential stability in the dual-phase-lag heat conduction theory, J.NonEquilibrium Thermodynamics, 27(2002), pp. 217-227.

[8] R. Quintanilla, A condition on the delay parameters in the one-dimensional dual-phase-lag thermoelastic theory, J. Thermal Stresses, 26(2003), pp. 713-721.

[9] R. Quintanilla, R. Racke, Qualitative aspects in dual-phase-lag thermoelasticity, SIAM Jour. Appl. Math., Accepted.

[10] M. Renardy, On the type of certain $C_{0}$-semigroups, Comm. PDE, 18(1993), pp. 1299-1307. 
[11] D. Y. Tzou, A unified approach for heat conduction from macro to micro-scales, ASME J. Heat Transfer, 117(1995), pp. 8-16.

[12] D. Y. Tzou, The generalized lagging response in small-scale and high-rate heating, Int. J. Heat Mass Transfer, 38 (1995), pp. 3231-3240.

[13] L.Wang, M. Mingtian, X. Zhou, Well-posedness and solution structure of dual-phaselagging heat conduction, Int. J. Heat Mass Transfer, 44 (2001), pp. 1659-1669.

[14] L.Wang, M. Mingtian, Well-posedness of dual-phase-lagging heat equation: higher dimensions, Int. J. Heat Mass Transfer, 45 (2002), pp. 1165-1171.

[15] M. Mingtian, L.Wang, Thermal oscillation and resonance in dual-phase-lagging heat conduction, Int. J. Heat Mass Transfer, 45 (2002), pp. 1055-1061. 